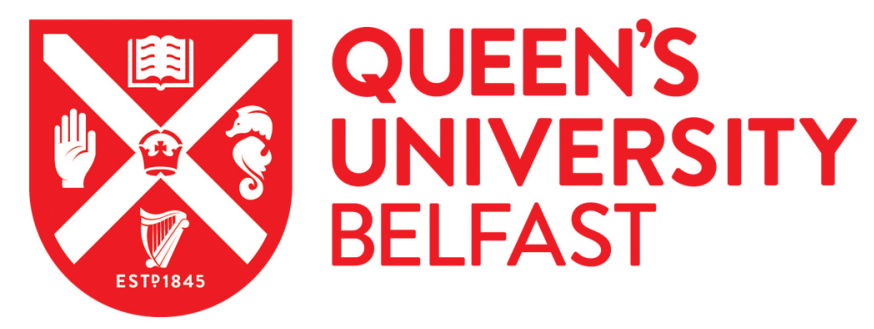

\title{
Boron in copper: A perfect misfit in the bulk and cohesion enhancer at a grain boundary
}

Lozovoi, A., \& Paxton, A. (2008). Boron in copper: A perfect misfit in the bulk and cohesion enhancer at a grain boundary. Physical Review B (Condensed Matter), 77(16), 165413-1-165413-14. [165413].

https://doi.org/10.1103/PhysRevB.77.165413

Published in:

Physical Review B (Condensed Matter)

Queen's University Belfast - Research Portal:

Link to publication record in Queen's University Belfast Research Portal

\section{General rights}

Copyright for the publications made accessible via the Queen's University Belfast Research Portal is retained by the author(s) and / or other copyright owners and it is a condition of accessing these publications that users recognise and abide by the legal requirements associated with these rights.

Take down policy

The Research Portal is Queen's institutional repository that provides access to Queen's research output. Every effort has been made to ensure that content in the Research Portal does not infringe any person's rights, or applicable UK laws. If you discover content in the Research Portal that you believe breaches copyright or violates any law, please contact openaccess@qub.ac.uk. 


\title{
Boron in copper: A perfect misfit in the bulk and cohesion enhancer at a grain boundary
}

\author{
A. Y. Lozovoi and A. T. Paxton \\ Atomistic Simulation Centre, School of Mathematics and Physics, Queen's University Belfast, \\ Belfast BT7 1NN, Northern Ireland, United Kingdom
}

(Received 14 November 2007; revised manuscript received 19 February 2008; published 10 April 2008)

\begin{abstract}
Using first principles electronic structure methods, we calculate the effects of boron impurities in bulk copper and at surfaces and grain boundaries. We find that boron segregation to the $\Sigma 5(310)[001]$ grain boundary should strengthen the boundary up to $1.5 \mathrm{ML}$ coverage $\left(15.24 \mathrm{at} . / \mathrm{nm}^{2}\right)$. The maximal effect is observed at $0.5 \mathrm{ML}$ and corresponds to boron atoms filling exclusively grain boundary interstices. In copper bulk, B causes significant distortion both in interstitial and regular lattice sites, for which boron atoms are either too big or too small. The distortion is compensated to a large extent when the interstitial and substitutional boron combine together to form a strongly bound dumbbell. Our prediction is that bound boron impurities should appear in a sizable proportion if not dominate in most experimental conditions. A large discrepancy between calculated heats of solution and experimental terminal solubility of $\mathrm{B}$ in $\mathrm{Cu}$ is found, indicating either a significant failure of the density functional approach or, more likely, strongly overestimated solubility limits in the existing $\mathrm{B}-\mathrm{Cu}$ phase diagram.
\end{abstract}

DOI: 10.1103/PhysRevB.77.165413

\section{INTRODUCTION}

Boron has an extremely good record in improving intergranular cohesion in metals. It is mostly famous for curing the long standing problem of room temperature brittleness in $\mathrm{Ni}_{3} \mathrm{Al} .{ }^{1}$ Boron segregation was found to reinforce grain boundaries in other intermetallic compounds (FeAl, NiAl, and $\mathrm{Ni}_{3} \mathrm{Si}$ ) and to improve low temperature ductility in bcc iron and refractory metals, such as Mo and W (see, e.g., Ref. 2 and references therein).

The effect of boron addition on copper is far less studied. According to the $\mathrm{Cu}-\mathrm{B}$ phase diagram, ${ }^{3}$ boron solubility in copper is low, i.e., 0.06 at. \% at room temperature rising to 0.29 at. \% at a eutectic temperature of $1013{ }^{\circ} \mathrm{C}$. Dissolved boron has a strong propensity to segregate to surfaces and interfaces. It is not clear whether segregation weakens or strengthens grain boundaries. Nevertheless, doping copper with boron is found to be efficient in preventing segregation of antimony to grain boundaries. ${ }^{4} \mathrm{~A}$ substantial improvement of mechanical properties of nanocrystalline $\mathrm{Cu}$ samples is reported as B segregation can be used to limit grain growth during heat treatment. ${ }^{5}$ Despite such encouraging experimental findings, quite surprisingly, no theoretical simulations of boron at copper grain boundaries or even free surfaces are known to us.

Similarly little is known about boron in bulk copper. It is not even clear whether boron occupies interstitial or substitutional positions. An analysis of boron's neighbors in the Periodic Table does not rule out either possibility. Carbon is an interstitial impurity in $\mathrm{Cu},{ }^{6}$ whereas $\mathrm{Al}$ and $\mathrm{Be}$ are substitutional impurities. In recent experimental work, in which accelerated boron ions were implanted in $\mathrm{Cu}$, both types of impurities were observed. ${ }^{7}$

In the present study, we employ standard density functional calculations to study the behavior of boron impurities at a copper grain boundary and in the bulk. We find that boron strengthens the $\Sigma 5\{310\}[001]$ symmetric tilt grain boundary in the whole range of boundary coverages investigated (up to $1.5 \mathrm{ML}$ ). The maximum strengthening occurs at $0.5 \mathrm{ML}$, at which boron exclusively occupies grain boundary interstices. We further identify mechanisms responsible for grain boundary strengthening within the framework of the "ghost impurity cycle" proposed in our previous work. ${ }^{8}$ The cycle admits the occupation of both substitutional and interstitial positions by impurity atoms at the interface, a feature that is fully exploited in the present study.

Due to the peculiar interplay of atomic sizes, interstitial and substitutional positions are equally unwelcoming to boron in bulk $\mathrm{Cu}$. Boron is too big an interstitial impurity and too small a substitutional impurity. As a result, both have nearly the same heat of solution, with the interstitial position marginally preferred. If, however, $\mathrm{B}$ atoms combine in dumbbells, then most of the elastic distortion of the host is eliminated, and a significant decrease in the heat of solution is achieved. The energy gain is so large that boron dumbbells should persist up to high temperatures. Another surprising finding of our study is a large disagreement between the theoretical heat of solution and the experimentally observed maximal solubility of $\mathrm{B}$ in $\mathrm{Cu}$.

This paper is organized as follows. Section II outlines definitions and thermodynamical relations used in the present work. The computational setup is described in Sec. III. The results on $\mathrm{B}$ in bulk $\mathrm{Cu}$ are presented in Sec. IV, whereas Sec. $\mathrm{V}$ is concerned with the effect of boron at the grain boundary. In the latter section, we first look at the change of the work of separation and at the impurity segregation energies (Sec. V A). Then, we discuss the atomic structure of the boundaries and free surfaces with different boron contents (Sec. V B). Finally, we apply the ghost impurity cycle to reveal the mechanisms responsible for the cohesion enhancement (Sec. V C). Our main findings are summarized in Sec. VI. A thermodynamic model used to estimate the concentration of different kinds of boron impurity in bulk copper is outlined in the Appendix.

\section{WORK OF SEPARATION AND GHOST IMPURITY CYCLE}

The energy release rate (the minimal energy per unit area of crack advance) associated with brittle cleavage of a grain 
boundary, $\mathcal{G}_{\text {cleav }}$, is the central quantity characterizing the resistance of the boundary to decohesion in the Rice-ThomsonWang approach. ${ }^{9,10}$ If $\mathcal{G}_{\text {cleav }}$ is lower than the energy release rate associated with emitting one dislocation $\mathcal{G}_{\text {disl }}$, then cracks remain atomically sharp and the crystal breaks in a brittle manner. If $\mathcal{G}_{\text {cleav }}>\mathcal{G}_{\text {diss }}$, the crack blunts and the crystal is ductile. Impurity segregation to grain boundaries can either decrease or increase $\mathcal{G}_{\text {cleav }}$. $\mathrm{Bi}$ in $\mathrm{Cu}$ is a classic example of the former. Copper grain boundaries with bismuth segregating eventually reach the condition $\mathcal{G}_{\text {cleav }}<\mathcal{G}_{\text {disl }}$ leading to a ductile-to-brittle transition.

In the limit of fast separation, which we assume in the present study, any impurity exchange between newly formed surfaces and bulk during the decohesion is prevented. In this limit, $\mathcal{G}_{\text {cleav }}$ can be identified with the reversible work of separation,

$$
\mathcal{G}_{\text {cleav }}=W_{\text {sep }}=\frac{1}{A}\left\{G^{s}-G^{\mathrm{gb}}\right\},
$$

where $A$ is the surface area, $G^{\mathrm{gb}}$ is the excess Gibbs free energy of a representative piece of material containing grain boundary, and $G^{s}$ is the sum of two excess Gibbs free energies corresponding to surfaces formed after decohesion. Equation (1) assumes that the impurity excess $\Gamma$ at the grain boundary is equal to the sum of surface impurity excesses. The excess in Eq. (1) is defined with respect to the underlying bulk crystal.

Our $a b$ initio calculations refer to the zero temperature limit; hence, the Gibbs free energies are replaced with total energies. It is convenient then to express the changes in $W_{\text {sep }}$ due to impurity in terms of segregation energies per impurity atom, $E_{\text {seg. }}$. (Segregation energy is the energy required to remove all impurity from an interface and distribute it in the bulk). Equation (1) becomes [see Eq. (6) in Ref. 8]

$$
W_{\text {sep }}(\mathbf{B})=W_{\text {sep }}(\mathbf{A})+\Gamma\left\{E_{\text {seg }}(\mathbf{B})-E_{\text {seg }}\left(\mathbf{B}_{s}\right)\right\},
$$

where $\mathbf{A}$ and $\mathbf{B}$ denote a pure material and a material with a segregant, respectively. Segregation energies are easy to obtain in an ab initio supercell approach using the total energies of supercells containing grain boundary (or surface) with and without impurity $\left[E_{\text {tot }}(\mathbf{B})\right.$ and $\left.E_{\text {tot }}(\mathbf{A})\right]$ and the same combination for the bulk $\left[E_{\mathrm{tot}}^{\mathrm{b}}(\mathbf{B})\right.$ and $\left.E_{\mathrm{tot}}^{\mathrm{b}}(\mathbf{A})\right]$. For a grain boundary, for example, we have

$$
E_{\mathrm{seg}}(\mathbf{B})=\frac{1}{N^{\mathrm{gb}}}\left\{E_{\mathrm{tot}}^{\mathrm{gb}}(\mathbf{B})-E_{\mathrm{tot}}^{\mathrm{gb}}(\mathbf{A})\right\}-\frac{1}{N^{b}}\left\{E_{\mathrm{tot}}^{\mathrm{b}}(\mathbf{B})-E_{\mathrm{tot}}^{\mathrm{b}}(\mathbf{A})\right\},
$$

where $N^{\mathrm{gb}}$ and $N^{\mathrm{b}}$ denote the number of impurity atoms included in grain boundary and bulk supercells, respectively. Equation (3) conveys a simple picture in which an impurity atom at the boundary is exchanged with a host atom in the bulk; if interstitial positions are involved, then Eq. (3) should be augmented by adding or subtracting a suitable amount of chemical potentials of the host atoms, which, again, are the total energies per atom in pure bulk.

Implicit in Eq. (3) is the fact that the bulk is sufficiently dilute so that neither interface nor bulk supercells include any additional randomly distributed impurity atoms. In the

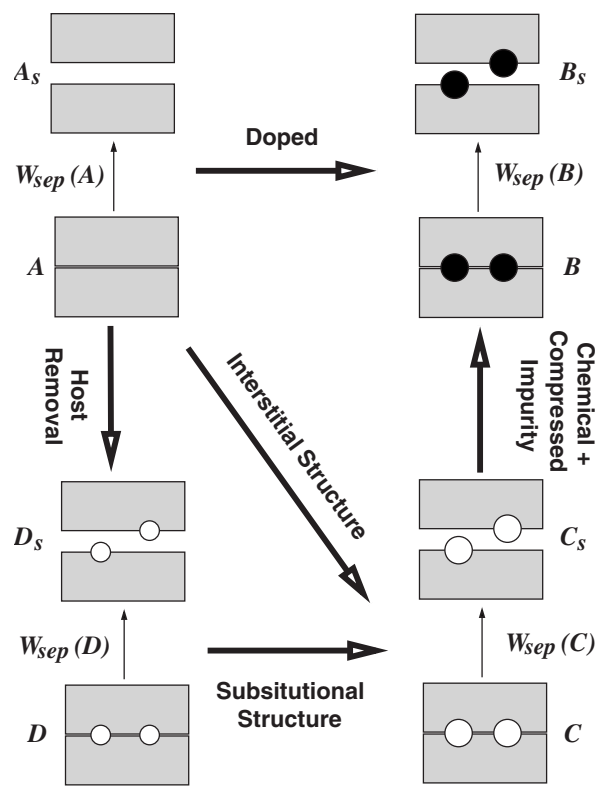

FIG. 1. Ghost impurity cycle used for the discussion of the effect of impurity on the grain boundary strength. A and $\mathbf{B}$ denote the pure and segregated grain boundaries, respectively, in their equilibrium geometry. Boundary $\mathbf{C}$ is created by substituting impurity atoms in B (black spheres) with vacancies (white spheres) without further atomic relaxation. Configuration $\mathbf{D}$ is $\mathbf{A}$ in which the host atoms that will be replaced by impurity in $\mathbf{B}$ are removed, with other atoms kept in place. $\mathbf{A}_{s}$ and $\mathbf{B}_{s}$ denote the free surfaces into which grain boundaries $\mathbf{A}$ and $\mathbf{B}$ cleave. $\mathbf{C}_{s}$ and $\mathbf{D}_{s}$ are prepared from $\mathbf{B}_{s}$ and $\mathbf{C}_{s}$, respectively, using the above strategy, namely, impurity atoms in $\mathbf{C}_{s}$ or host atoms to be replaced by impurity in $\mathbf{D}_{s}$, are removed, keeping the positions of other atoms fixed. Path $\mathbf{A} \rightarrow \mathbf{D} \rightarrow \mathbf{C} \rightarrow \mathbf{B}$ refers to substitutional impurities, whereas path $\mathbf{A} \rightarrow \mathbf{C} \rightarrow \mathbf{B}$ applies to interstitial impurities (Ref. 8).

dilute limit, the grain boundary impurity excess $\Gamma$ and excess volume $v^{\mathrm{xs}}$ per unit area can be found as

$$
\begin{gathered}
\Gamma=\frac{N^{\mathrm{gb}}}{A}, \\
v^{\mathrm{xs}}=\frac{1}{A}\left\{V(\mathbf{B})-N_{0}^{\mathrm{gb}} \Omega_{0}\right\},
\end{gathered}
$$

where $V(\mathbf{B})$ is the volume of a relaxed grain boundary supercell with impurity, $N_{0}^{\mathrm{gb}}$ is the number of host atoms in this supercell, and $\Omega_{0}$ is the atomic volume in pure bulk.

To separate various aspects of grain boundary weakening or strengthening by impurity atoms originating from their size, positions, and chemical identity, the ghost impurity cycle introduced in Ref. 8 and shown in Fig. 1 is rather useful. In this cycle, the direct transition from unsegregated to segregated state $\mathbf{A} \rightarrow \mathbf{B}$ is replaced with a gedanken path through intermediate configurations $\mathbf{A} \rightarrow \mathbf{D} \rightarrow \mathbf{C} \rightarrow \mathbf{B}$ for both grain boundary and surfaces, and the respective changes in $W_{\text {sep }}$ are evaluated. Configuration $\mathbf{C}$ is created from $\mathbf{B}$ by removing all impurity atoms without subsequent relaxation. These missing atoms are referred to as "ghosts" since they create forces that keep host atoms in place but do not con- 
tribute to the energy of the system in any other way. Such ghosts are distorted vacant sites for substitutional impurities or centers of expansion for interstitial impurities. Configuration $\mathbf{D}$ is constructed from $\mathbf{A}$ in a similar way, except that we remove the host atoms defined by impurity sites in $\mathbf{B}$ only if these impurities replace host atoms. For interstitial impurities, configuration $\mathbf{D}$ is not visited (see interstitial path in Fig. $1)$.

The same approach is used for the generation of surface configurations $\mathbf{A}_{s}-\mathbf{D}_{s}$. $\mathbf{B}_{s}$ and $\mathbf{A}_{s}$ represent the equilibrium geometry of free surfaces with and without impurity, respectively. Configuration $\mathbf{C}_{s}$ is created from $\mathbf{B}_{s}$ by removing impurity atoms, whereas in $\mathbf{D}_{s}$ one removes only those host atoms that will be replaced with impurity in $\mathbf{B}_{s}$. The remaining atoms in $\mathbf{C}_{s}$ and $\mathbf{D}_{s}$ are not allowed to move in response to the removal of some of their neighbors. Note that the impurity atoms occupying substitutional positions at a grain boundary can be interstitial impurities at free surfaces (or vice versa). In terms of Fig. 1, this would mean that the substitutional path should be used for grain boundaries, whereas the interstitial path should be taken for surfaces. More generally, one could envisage a situation in which part of impurity atoms occupy interstices, whereas the another part substitute host atoms. We shall shortly describe how to deal with such situations.

As argued in Ref. 8, the change of $W_{\text {sep }}$ at the $\mathbf{A} \rightarrow \mathbf{D}$ step describes grain boundary weakening due to some host-host bonds being broken ["host removal" (HR) mechanism]. Transition $\mathbf{D} \rightarrow \mathbf{C}$ corresponds to the distortion of the atomic structure of pure boundary and surface caused by impurity ["substitutional structure" (SS) mechanism]. Finally, step C $\rightarrow \mathbf{B}$ brings in the impurity-host chemical interactions and, if relevant, associated changes in neighboring host-host bonds. For oversized impurity atoms, this step also incorporates the elastic energy stored in compressed impurity atoms. These two mechanisms cannot be separated; hence, we refer to this step as a "chemical+compressed impurity" (CC) mechanism. For more details regarding the cycle and its implementation, the reader is referred to Ref. 8.

The ghost impurity cycle outlined above treats interstitial and substitutional impurities on an equal basis. This is vital for the purposes of the present study in which we shall be introducing boron into substitutional and interstitial positions at a grain boundary, sometimes even simultaneously. The way to deal with the "coexistence" of the substitutional and interstitial paths in Fig. 1 is to formally include configuration D into the latter, making it indistinguishable from A. In such case, Eq. (2) can be used without making any specific allowances. The same applies to surfaces with impurities occupying adatom positions. These can be treated similar to interstitial impurities at grain boundaries.

\section{CALCULATION DETAILS}

The $\Sigma 5\{310\}[001]$ tilt grain boundary is represented in our study by a periodic supercell containing two grain boundaries with opposite orientations, without any vacuum. Altogether, there are 38 atoms in the supercell, with each atom representing one $\{310\}$ layer, except the grain boundary

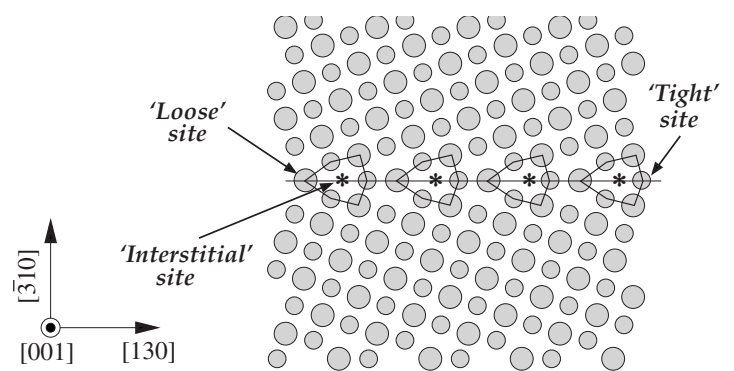

FIG. 2. Equilibrium structure of the $\Sigma 5\{310\}[001]$ symmetric tilt grain boundary in pure copper. Larger and smaller circles represent alternating (001) $\mathrm{Cu}$ planes. The grain boundary plane contains two inequivalent $\mathrm{Cu}$ atoms which we refer to as the loose and tight sites. The interstitial site suitable for segregation of small impurity atoms is shown with an asterisk.

plain that contains two atoms, i.e., the "tight" and the "loose" sites. These two sites and an "interstitial" site shown in Fig. 2 are considered as three possible segregation sites for B. Occupation of any one, any two, or all three of these sites corresponds to $0.5,1$, and $1.5 \mathrm{ML}$ coverages in our notation. As we shall see in Sec. V B, severe atomic relaxation of the boundary involving lateral translation of the grains may significantly change the local environment of segregated atoms. We shall therefore label configurations with respect to positions in which the impurity was initially placed.

To represent free (310) surfaces, we use the same supercell with 25 layers of copper, the rest being vacuum. Outermost copper layers are replaced with impurity layers if we need to model a segregated surface. The situation in which a grain boundary containing 0.5 or $1.5 \mathrm{ML}$ of impurity cleaves into surfaces with even amount of impurity requires us to double the supercell along the [001] direction. Cubic supercells containing up to 108 atoms $(3 \times 3 \times 3 \mathrm{fcc}$ cells $)$ were used to model boron impurities in bulk $\mathrm{Cu}$.

Our first principles calculations employed the full potential linear muffin tin orbital (FP LMTO) method, as implemented in the NFP code..$^{20}$ Calculations were semirelativistic, without spin polarization. We used the local density approximation (LDA) in the parametrization of von Barth and Hedin, ${ }^{21}$ which was modified by Moruzzi et al. ${ }^{22}$ Other parameters ( $k$-point meshes, real space meshes, etc.) were the same as in Ref. 8 to which the reader is referred for further details.

\section{BORON IMPURITY IN BULK COPPER}

\section{A. Pure boron}

Solid boron can exist in a number of relatively stable allotropic modifications-rhombohedral, tetragonal, and even amorphous. It is not clear which phase corresponds to the ground state of B at ambient conditions. Two rhombohedral phases, $\alpha$ - and $\beta$-B are the most likely candidates. $\alpha$-B becomes unstable at $1200{ }^{\circ} \mathrm{C}$ and converts to $\beta$-B at above $1500{ }^{\circ} \mathrm{C}$, but $\beta$-B does not transform back to $\alpha$-B upon cooling (see Ref. 14 and references therein). Hence, kinetic effects must impose severe limitations in this material. $\alpha$-B 
TABLE I. Ground-state properties of $\alpha$-B: equilibrium volume $V_{0}$, rhombohedral lattice constant $a_{0}$, rhombohedral angle $\phi$, internal coordinates of boron atoms, $x_{1}, z_{1}, x_{2}$, and $z_{2}$, bulk modulus $B$, and the energy difference (per atom) between fcc and $\alpha$ boron, $\Delta E_{\mathrm{fcc}}$, obtained in the present study and other $a b$ initio calculations. Experimental data in the last column are from Ref. 11 unless stated otherwise.

\begin{tabular}{|c|c|c|c|c|c|c|c|c|c|c|c|c|}
\hline $\begin{array}{l}\text { Method } \\
\text { (LDA or GGA) }\end{array}$ & $\begin{array}{l}\text { FP LMTO } \\
\text { (LDA) }\end{array}$ & $\begin{array}{l}\text { PAW } \\
\text { (LDA) }\end{array}$ & $\begin{array}{l}\text { PW-PP } \\
\text { (LDA) }\end{array}$ & $\begin{array}{l}\text { LMTO } \\
\text { (LDA) }\end{array}$ & $\begin{array}{l}\text { FP LMTO } \\
\text { (LDA) }\end{array}$ & $\begin{array}{l}\text { PW-PP } \\
\text { (LDA) }\end{array}$ & $\begin{array}{l}\text { PW-PP } \\
\text { (LDA) }\end{array}$ & $\begin{array}{l}\text { PW-PP } \\
\text { (LDA) }\end{array}$ & $\begin{array}{l}\text { PW-PP } \\
\text { (LDA) }\end{array}$ & $\begin{array}{l}\text { US-PP } \\
\text { (GGA) }\end{array}$ & $\begin{array}{l}\text { US-PP } \\
\text { (GGA) }\end{array}$ & Expt. \\
\hline Reference & \multicolumn{2}{|c|}{ Present study } & \multicolumn{2}{|c|}{ Ref. 12} & \multicolumn{2}{|c|}{ Ref. 13} & Ref. 14 & Ref. 15 & Ref. 16 & Ref. 17 & Ref. 18 & Ref. 11 \\
\hline$V_{0}\left(\AA^{3}\right)$ & 6.899 & 6.993 & 7.05 & 6.93 & 7.06 & 6.88 & & & & 6.946 & 7.30 & $7.337^{\mathrm{a}}$ \\
\hline$a_{0}(\AA)$ & 4.967 & 4.989 & & & & & 5.034 & 4.98 & 4.967 & 4.973 & & \\
\hline$\phi(\mathrm{deg})$ & 58.055 & 58.063 & & & & & 58.119 & 58.2 & 58.65 & & & 58.06 \\
\hline$x_{1}$ & 0.0106 & 0.0105 & & & 0.010 & & & & & & & 0.0104 \\
\hline$z_{1}$ & -0.3460 & -0.3458 & & & -0.344 & & & & & & & -0.3427 \\
\hline$x_{2}$ & 0.2211 & 0.2215 & & & 0.220 & & & & & & & 0.2206 \\
\hline$z_{2}$ & -0.3694 & -0.3700 & & & -0.369 & & & & & & & -0.3677 \\
\hline$B(\mathrm{GPa})$ & 232 & & 249 & 266 & 230 & 227 & & & 218.4 & & & $224^{\mathrm{a}}$ \\
\hline$\Delta E_{\mathrm{fcc}}(\mathrm{eV})$ & 1.30 & 1.35 & 1.43 & 1.83 & 1.31 & 1.39 & & & & & & \\
\hline
\end{tabular}

${ }^{\mathrm{a}}$ Reference 19.

was found lower in energy by $0.036 \mathrm{eV} /$ atom in Ref. 16 and by $0.283 \mathrm{eV} /$ atom in Ref. 17 , and is assumed to represent the ground state in the present study.

The unit cell of the rhombohedral $\alpha$-B consists of 12 atoms forming an icosahedron. The equilibrium structure, bulk modulus, and fcc- $\alpha$ energy difference obtained in our study are in good agreement with other calculations and show the usual discrepancies with experiment associated with the LDA, namely, underestimation of atomic volumes and consequent overestimation of the bulk moduli (see Table I). Calculations are scalar relativistic, are fully relaxed, and employ the $8 \times 8 \times 8$ Monkhorst-Pack mesh of $k$ points. Increasing the $k$-point mesh to $12 \times 12 \times 12$ changes the total energy by less than $10^{-5} \mathrm{Ry}$, whereas the forces remain within the convergence criterion of $10^{-3} \mathrm{Ry} / \mathrm{bohr}$ used throughout the whole study.

\section{B. Copper-boron solid solution}

As noted in the Introduction, the available observations do not allow one to unambiguously conclude whether the ground state ${ }^{23}$ of boron in bulk $\mathrm{Cu}$ is interstitial $\mathrm{B}_{i}$ or substitutional $\mathrm{B}_{s}$. We calculated the heats of solution of both impurity types using 32 and 108 atom supercells and find that $\mathrm{B}_{i}$ (in octahedral site) is marginally more stable (by $0.04 \mathrm{eV}$ with a 108 atom supercell, Table II). The difference is small; hence, it seems reasonable to expect that both $\mathrm{B}_{i}$ and $\mathrm{B}_{s}$ can be found at elevated temperatures, as was indeed observed. $^{7,24,25}$

Also included in the table are the activation energies $E_{a}$ for boron diffusion. Normally, one would expect the latter to be dominated by the interstitial mechanism, which experimental results apparently confirm. ${ }^{7}$ The calculated activation energy for interstitial diffusion $(0.93 \mathrm{eV})$ is somewhat higher than the experimental value $(0.57 \mathrm{eV})$ but is still in reasonable agreement. Interestingly, it is suggested ${ }^{24}$ that the diffusion of substitutional boron occurs by means of a direct exchange with copper atoms rather than by a vacancy mechanism. We leave the theoretical verification of this im- portant result for future studies, not least because the situation with impurity defects themselves appears more complex than just being a simple combination of interstitial and substitutional boron atoms.

Inspection of dilation volumes $\Omega_{d}$ in Table II reveals that either the insertion of a boron atom into an interstice or the replacement of a host atom at a regular lattice site leads to a

TABLE II. Boron in bulk $\mathrm{Cu}$ : enthalpy of solution $H_{s}$ (per impurity atom) and the relative dilation volume $\Omega_{d} / \Omega_{0}$ of interstitial boron $\mathrm{B}_{i}$, substitutional boron $\mathrm{B}_{s}$, and boron dumbbells $\mathrm{B}_{d}$ with different orientations. $\Delta H_{s}$ is the enthalpy relative to that of the $s\langle 100\rangle$ dumbbell (per entity). $N_{a t}$ denotes the number of lattice sites in a supercell used in the calculation, $\Omega_{d}$ is the change of the volume of this supercell when either a single impurity $\left(\mathrm{B}_{i}\right.$ or $\left.\mathrm{B}_{s}\right)$ or a dumbbell $\mathrm{B}_{d}$ is introduced, $\Omega_{0}$ is the atomic volume in pure fcc $\mathrm{Cu}$, and $E_{a}$ is the activation energy for impurity diffusion. Experimental data on $E_{a}$ are from Ref. 7. Theoretical results are obtained by the FP LMTO method unless indicated otherwise.

\begin{tabular}{lccccc}
\hline \hline Impurity & $N_{a t}$ & $\begin{array}{c}H_{s} \\
(\mathrm{eV})\end{array}$ & $\begin{array}{c}\Delta H_{s} \\
(\mathrm{eV})\end{array}$ & $\Omega_{d} / \Omega_{0}$ & $\begin{array}{c}E_{a} \\
(\mathrm{eV})\end{array}$ \\
\hline $\mathrm{B}_{i}$ & 32 & 1.58 & -0.10 & 0.88 & 1.31 \\
$\mathrm{~B}_{i}{ }^{\mathrm{a}}$ & 32 & 1.63 & & 0.93 & \\
$\mathrm{~B}_{i}$ & 108 & 1.66 & 0.06 & 0.89 & 0.93 \\
$\mathrm{~B}_{s}$ & 32 & 1.70 & 0.02 & -0.44 & \\
$\mathrm{~B}_{s}$ & 108 & 1.70 & 0.10 & -0.45 & \\
$\mathrm{~B}_{d}:$ & & & & & \\
$a\langle 111\rangle$ & 32 & 1.69 & 1.70 & 0.52 & \\
$s\langle 111\rangle$ & 32 & 1.04 & 0.40 & 0.38 & \\
$a\langle 100\rangle^{\mathrm{b}}$ & 32 & & & & \\
$s\langle 100\rangle$ & 32 & 0.84 & 0 & 0.25 & \\
$s\langle 100\rangle$ & 108 & 0.80 & 0 & 0.24 & \\
Expt. & & & & & $\mathrm{B}_{i}: 0.57(5)$ \\
& & & & & \\
\hline \hline
\end{tabular}

aPAW calculations.

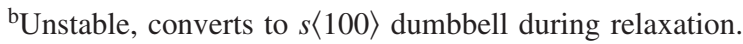


significant volume change, which is negative for $\mathrm{B}_{s}$ and positive for $\mathrm{B}_{i}$. Hence, one could hypothezise that combining $\mathrm{B}_{s}$ and $\mathrm{B}_{i}$ into a dimer might eliminate most of the elastic distortion of the lattice. To explore this idea, we repeated the calculation with $\mathrm{B}_{i}$ and $\mathrm{B}_{s}$ placed next to each other either along the $\langle 111\rangle$ or the $\langle 100\rangle$ direction. In addition, we also considered two boron atoms symmetrically arranged around a vacant site along the same directions. We shall refer to the former and the latter as asymmetric and symmetric dumbbells, respectively. Among these, the symmetric $\langle 100\rangle$ dumbbell, $s\langle 100\rangle$, appears to be the most stable (see Table II). The heat of solution of the $s\langle 100\rangle$ dumbbell is a factor of 2 lower than those of the single impurities, indicating that the dumbbells should dominate at low temperatures and even survive up to the eutectic temperature $T_{e}=1013{ }^{\circ} \mathrm{C}$.

To verify the last point, we use a simple model in which boron dumbbells $\mathrm{B}_{d}$ as well as single impurities $\mathrm{B}_{i}$ and $\mathrm{B}_{s}$ are treated as three types of coexisting point defects forming an ideal solution. The model is described in the Appendix, together with the results obtained within this model in the dilute limit. We find, in particular, that the concentration of dumbbells exceeds those of single impurities in most conditions unless the temperature is close to $T_{e}$ or the boron content is very small. Otherwise, all three impurity forms coexist, both in the single phase and the two phase regions of the $\mathrm{Cu}-\mathrm{B}$ phase diagram, with the dumbbells usually being the dominant kind.

In fact, this can be anticipated already from the difference of the solution enthalpies per entity $\left(H_{s}\right.$ for single impurities and $2 H_{s}$ for dumbbells) listed in Table II in the column for $\Delta H_{s}$. These differences serve to estimate the relative amount of defects at terminal solubility [see Eq. (A15) in the Appendix].

The fact that adding $\mathrm{B}$ to $\mathrm{Cu}$ gives rise to three types of competing defects is due to a remarkable coincidence that B atoms are so perfectly "incompatible" with the $\mathrm{Cu}$ lattice that the interstitial and substitutional sites are nearly degenerate in energy; furthermore, boron dumbbells stabilized by this misfit strain turn out to have almost the same heat of solution per dumbbell as those of the single impurities per atom.

The individual concentrations of the defects might, of course, change if temperature effects, such as atomic vibrations and lattice expansion, are fully taken into account. In addition, the association of several (three, four, etc.) impurities can also play a role, at least at low temperatures. Nevertheless, we believe that our results provide a strong indication that in equilibrium copper-boron alloys, a significant fraction of the impurities are found in "bound" states.

We are not aware of any metallic system in which dilute impurity would aggregate. Interestingly, Dewing ${ }^{26}$ noted that activity measurements of $\mathrm{B}$ in molten $\mathrm{Cu}$ suggested that $\mathrm{B}$ should dimerize in dilute solutions. Here, we seem to arrive at the same conclusion although for different reasons as elastic strain does not exist in liquids. Our finding might still be relevant to the processing of the experimental data on copper-boron melts as the solid alloy in these studies is customarily assumed to be an ideal ${ }^{27}$ or regular ${ }^{28}$ solution of fully dissociated impurity atoms.

Another observation that stems from the heats of solution in Table II is the fact that the solubility limits indicated in the experimental phase diagram, ${ }^{3}$ i.e., 0.06 at. $\%$ at room temperature and 0.29 at. $\%$ at $T_{e}$, are much too high in comparison with the enthalpies that we obtained. Assuming that impurity atoms are in the form of dimers and that boron precipitates as the pure rhombohedral $\alpha$-phase, the above solubilities would translate into the Gibbs free energies of solution of $0.42 \mathrm{eV} /$ atom at $T_{e}$ and $0.12 \mathrm{eV} /$ atom at room temperature. Assuming single impurities would result in an even larger disagreement. Using the aforementioned ideal solution model, together with the heats of solution in Table II, leads to a 3 order of magnitude discrepancy in terminal solubility at $T_{e}$, which increases to more than 20 orders of magnitude at room temperature (see the Appendix). Temperature effects can be noticeable at $T_{e}$ but cannot explain the discrepancy at room temperature.

Puzzled with this inconsistency, we compared our FP LMTO calculations to those by the projected augmented wave (PAW) method ${ }^{29,30}$ as implemented in the VASP code. ${ }^{31,32} H_{s}$ obtained for $\mathrm{B}_{i}$ in the 32 atom cell $(1.63 \mathrm{eV})$ is indeed very close to the FP LMTO result $(1.58 \mathrm{eV}$ ) (see Table II). Hence, we conclude that the heats of solution presented in Table II are the correct LDA result. Furthermore, in order to make sure that it is not LDA itself that leads to the discrepancy, we repeated the above calculation using the generalized gradient approximation (GGA) with the PerdewBurke-Ernzerhof exchange-correlation functional ${ }^{33}$ and arrived at $H_{s}$ as large as $1.49 \mathrm{eV}$.

The B-Cu phase diagram in Ref. 3 is taken from the critical assessment of available experimental data from Chakrabarti and Laughlin, ${ }^{34}$ in which the data on maximal solubility of $\mathrm{B}$ in $\mathrm{Cu}$ are solely based on work by Smiryagin and Kvurt. ${ }^{35}$ The solubility limits mentioned above are those estimated in this latter study $(0.05$ and $0.01 \mathrm{wt}$. \% B translated into at. \%) and appear to serve more as an upper boundary rather than as exact numbers. Chakrabarti and Laughlin indeed commented in their assessment that "it is likely that the actual solubility is even lower than that given by [Ref. 35]." We expect it to be significantly lower and appeal to future experimental work to correct the terminal boron solubility in published $\mathrm{B}-\mathrm{Cu}$ phase diagrams.

\section{BORON AT A COPPER GRAIN BOUNDARY}

\section{A. Work of separation and grain boundary excess volume}

The work of separation of a grain boundary at given impurity excess is the difference in total energy between equivalent pieces of material containing the boundary and free surfaces into which the boundary cleaves. Both the grain boundary and the surface pieces should be taken in their lowest energy state.

The lowest energy grain boundary can be found by comparing the energies of relaxed grain boundaries with impurities initially placed into various sites (substitutional or interstitial). This procedure does not, of course, guarantee an arrival at the global minimum, but is a practical alternative to a full optimization of grain boundary structure and includes rigid translation of the grains.

The surfaces do not require translations, but a complication here is that one does not know in advance the optimal 


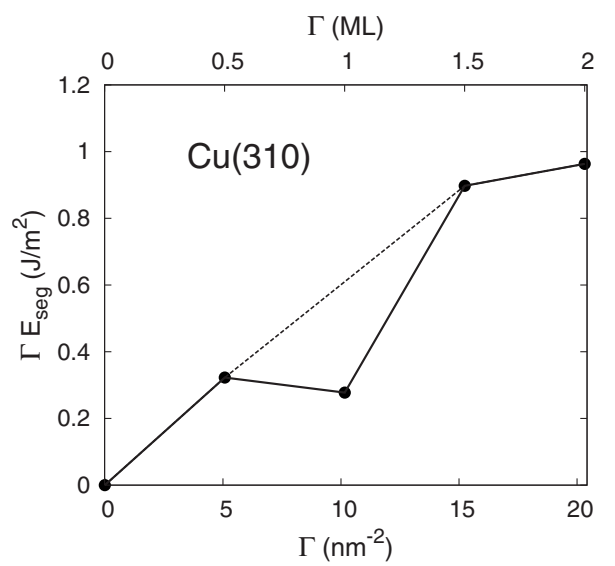

FIG. 3. Convex hull plot for B impurity at the $\mathrm{Cu}(310)$ surface. Circles correspond to the lowest energy surfaces for each coverage found in our study. According to these, the energy of the system is lowered if ordered unreconstructed $1 \mathrm{ML}$ surface decomposes into 0.5 ML and 1.5 surfaces (dashed line).

distribution of the impurity atoms between two newly created surfaces. Usually, the impurity splits equally, but not always. More generally, even if equal amounts of impurity are experimentally detected for a cleaved macroscopic sample, there still remains a possibility that the surfaces would contain patches with uneven impurity coverage.

The combination of surfaces that produces the lowest energy at given overall amount of impurity can be identified if one employs the convex hull construction. In Fig. 3, we plot the total segregation energy to a surface $G_{\mathrm{seg}}^{s}=\Gamma E_{\mathrm{seg}}^{s}$ as a function of impurity excess $\Gamma$. The quantity $G_{\text {seg }}^{s}$ shows how much the segregation decreases the energy of a piece of material containing a surface. Therefore, any concave region of the curve indicates that there exists a combination of surfaces which provide lower energy. In our case, we find that the 1 ML grain boundary splits into surfaces with coverages 0.5 and 1.5 ML, whereas the other boundaries cleave evenly.

The resulting works of separation are listed in Table III,

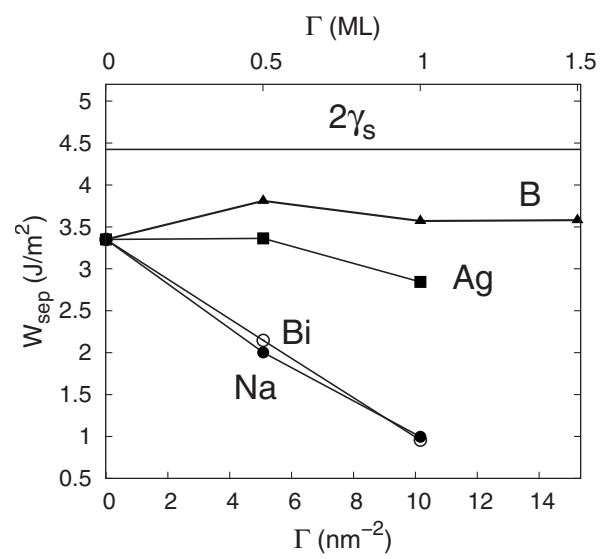

FIG. 4. The work of separation of the $\Sigma 5(310)[001] \mathrm{Cu}$ grain boundary, $W_{\text {sep }}$, as a function of impurity excess $\Gamma$. Data for $\mathrm{Bi}, \mathrm{Na}$, and $\mathrm{Ag}$ are from Ref. 8. The horizontal line corresponds to twice the surface energy of $\mathrm{Cu}(310)$ (Ref. 8), $\gamma_{s}=2.21 \mathrm{~J} / \mathrm{m}^{2}$, which provides a natural upper bound for $W_{\text {sep. }}$.

together with segregation energies and grain boundary excess volumes. The maximal $W_{\text {sep }}$ for each coverage correspond to the lowest energy grain boundary and are highlighted in bold. We thus find that at $0.5 \mathrm{ML}$ boron prefers the interstitial site, and at $1 \mathrm{ML}$ replacing $\mathrm{Cu}$ at both the loose and tight sites provides the best option. Corresponding works of separation are compared to those obtained for $\mathrm{Bi}, \mathrm{Na}$, and Ag (Ref. 8) in Fig. 4. Contrary to these latter impurities, boron increases $W_{\text {sep }}$ for the whole range of coverages considered. The largest strengthening effect is observed at 0.5 ML, at which $W_{\text {sep }}$ increases by $0.5 \mathrm{~J} / \mathrm{m}^{2}$ and becomes as large as $3.81 \mathrm{~J} / \mathrm{m}^{2}$. This can be compared to twice the surface energy of pure $\mathrm{Cu},{ }^{8} 2 \gamma_{310}^{s}=4.42 \mathrm{~J} / \mathrm{m}^{2}$, which provides an upper bound for $W_{\text {sep }}$ above which a grain boundary would become stronger than a bulk.

Grain boundary segregation energies in Table III favorably compare to experimental estimations of $0.4-0.5 \mathrm{eV},{ }^{4}$ especially for high coverages. These energies assume the

TABLE III. $\Sigma 5(310)$ [001] $\mathrm{Cu}$ grain boundary with B at various segregation sites: grain boundary excess volume per unit area, $v^{\mathrm{xs}}$, average segregation energies per impurity atom, $E_{\mathrm{seg}}$, and work of separation $W_{\text {sep }}$. Letters $l, t$, and $i$ in the second line correspond to impurity atoms being initially placed in the loose, tight, and interstitial positions at the grain boundary plane (see Fig. 2) and then relaxed. The optimal impurity distribution between cleaved surfaces is indicated in the last line. The quantities corresponding to the lowest energy grain boundaries at each coverage are highlighted in bold.

\begin{tabular}{|c|c|c|c|c|c|c|c|c|c|}
\hline \multirow{2}{*}{$\begin{array}{l}\text { Impurity excess (ML) } \\
\text { Site }\end{array}$} & \multirow[t]{2}{*}{0} & \multicolumn{3}{|c|}{0.5} & \multicolumn{3}{|c|}{1} & \multirow{2}{*}{$\frac{1.5}{l+t+i}$} & \multirow[t]{2}{*}{2} \\
\hline & & $l$ & $t$ & $i$ & $l+t$ & $l+i$ & $t+i$ & & \\
\hline $\begin{array}{l}\text { GB excess volume per unit } \\
\text { area, } v^{\text {ex }}(\AA)\end{array}$ & 0.28 & 1.02 & 0.53 & 0.45 & 0.87 & 0.78 & 0.66 & 0.98 & \\
\hline \multicolumn{10}{|l|}{ Segregation energy $E_{\mathrm{seg}}(\mathrm{eV})$ : } \\
\hline to the $(310)$ surface & 0 & 0.40 & 0.40 & 0.40 & 0.17 & 0.17 & 0.17 & 0.37 & 0.30 \\
\hline to the grain boundary & 0 & -0.66 & 0.40 & 0.96 & 0.51 & 0.31 & 0.29 & 0.46 & \\
\hline Work of separation $W_{\text {sep }}\left(\mathrm{J} / \mathrm{m}^{2}\right)$ & 3.35 & 2.49 & 3.35 & 3.81 & 3.57 & 3.24 & 3.21 & 3.58 & \\
\hline Cleavage mode & & \multicolumn{3}{|c|}{$0.5+0.5$} & \multicolumn{3}{|c|}{$0.25+0.75$} & $0.5+0.5$ & \\
\hline
\end{tabular}




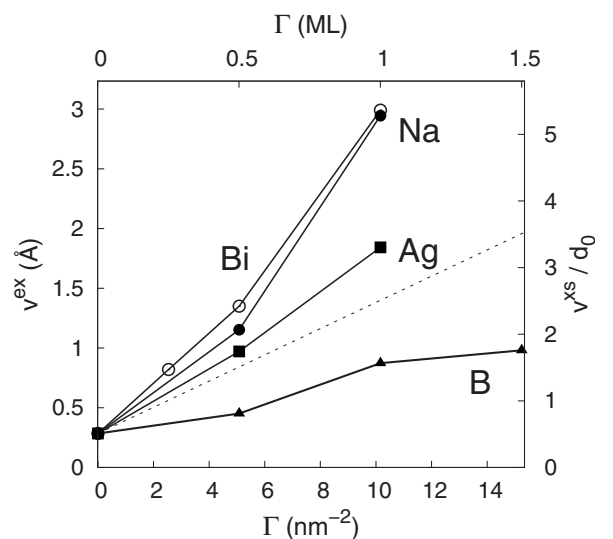

FIG. 5. The grain boundary excess volume per unit area, $v^{\mathrm{xs}}$, as a function of impurity excess $\Gamma$. Data for $\mathrm{Bi}, \mathrm{Na}$, and $\mathrm{Ag}$ are from Ref. 8. The right hand scale gives $v^{\mathrm{xs}}$ in units of $\{310\}$ interlayer spacing in bulk copper, $d_{0}=0.5576 \AA$. The dotted line corresponds to a hypothetical ideal impurity identical with $\mathrm{Cu}$ atoms.

$s\langle 100\rangle$ dumbbells to be the ground state of B in bulk $\mathrm{Cu}$ (see Table II). Had we used interstitial or substitutional B impurities instead, then the segregation energies would have been $0.8 \mathrm{eV}$ higher. We take this as an independent confirmation of the fact that $\mathrm{B}$ impurities in bulk $\mathrm{Cu}$ dimerize.

Grain boundary excess volumes $v^{\mathrm{xs}}$ in Table III are calculated using the dilute bulk limit [Eq. (5)]. They are smaller than those for $\mathrm{Bi}, \mathrm{Na}$, and $\mathrm{Ag}$, as shown in Fig. 5. The dotted line in Fig. 5 corresponds to the excess volume if $\mathrm{Cu}$ is notionally considered as an impurity (one could think of the ${ }^{65} \mathrm{Cu}$ isotope, for instance). The segregation of such "ideal" impurity leaves any grain boundary intact; hence, in accord with Eqs. (4) and (5), $v^{\mathrm{xs}}(\Gamma)$ is a straight line with the slope given by $\Omega_{0}$. The fact that the excess volumes of grain boundaries with B lie below the dotted line in Fig. 5 indicates a denser packing of atoms at the grain boundary with boron compared to the pure boundary.

\section{B. Atomic structure of the segregated surfaces and grain boundaries}

The atomic structure of relaxed grain boundaries with 0.5 ML of boron is relatively simple. The segregated boundaries retain the structure of the equilibrium pure boundary shown in Fig. 2 responding to the insertion of impurity by either minor shrinking (tight site) or expansion (loose and interstitial sites). These boundaries are not shown here.

The structures of grain boundaries with 1 and $1.5 \mathrm{ML}$ are more complex (see Fig. 6). The exception is the $t+i$ boundary (i.e., boron atoms segregate to the tight and interstitial sites), which does not significantly change compared to the pure boundary. However, the average segregation energy of boron to the $t+i$ boundary is lower than those for either $t$ or $i 0.5$ ML boundaries (see Table III).

Among the $1 \mathrm{ML}$ boundaries, the lowest energy corresponds to the more open $l+t$ boundary in which boron replaces copper at both loose and tight sites. This boundary experiences a large lateral shift of the grains. As a result of this shift, the boron atoms lying in adjacent (001) planes

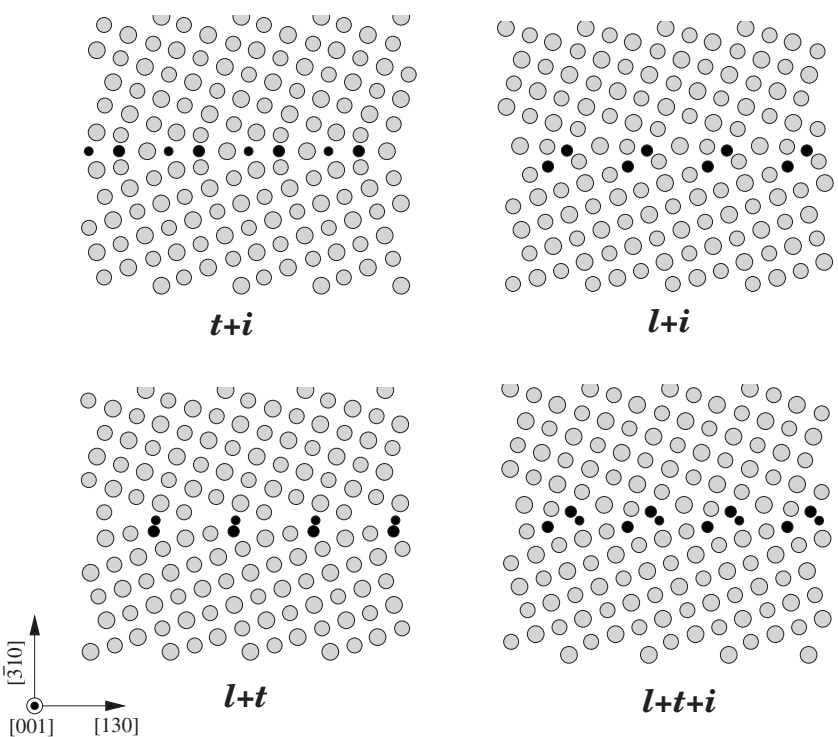

FIG. 6. Relaxed $\Sigma 5$ copper grain boundary with 1 and $1.5 \mathrm{ML}$ of boron. For 1.5 ML, boron occupies loose, tight, and interstitial sites at the grain boundary plane. For $1 \mathrm{ML}$, any two of them are occupied (three combinations). Works of separation and grain boundary excess volumes corresponding to these boundaries are listed in Table III. Larger and smaller circles correspond to host and impurity atoms lying in neighboring (001) planes.

become nearest neighbors and form boron "strings" running along the [001] direction (normal to the plane of the drawing in Fig. 6).

The $l+i$ boundary also entails a rigid translation of the grains. This time, however, boron atoms lie in the same plane and therefore cannot form [001] strings. The energy of the $l+i$ boundary is close to the energy of the $t+i$ boundary despite their atomic structures being very different. As a matter of fact, the $l+i$ boundary can be interpreted as the $i$ boundary in which the grain boundary plane is shifted normal to itself by one layer and another boron atom substitutes a copper atom in the adjacent plane.

The 1.5 ML $l+t+i$ boundary combines the features of the $t+i$ boundary (interstitial boron surrounded by six $\mathrm{Cu}$ atoms) and the $l+t$ boundary (boron strings). The average segregation energy for this boundary is close to that of the 1 ML $l$ $+t$ boundary.

The following observation is worthwhile here. A substantial fall-off of segregation energy with the amount of segregants appears to be a common feature of boron doped intermetallic compounds. As Lejček and Fraczkiewicz note, ${ }^{2}$ this could be formally described by either introducing a strong repulsive term into the Fowler-Guggenheim segregation isotherm or by using the standard Langmuir-McLean isotherm, but with a limited number of segregation sites. As we observe here, the former approach might be physically misleading, at least for the $\mathrm{Cu}-\mathrm{B}$ system. For instance, the 1 ML $l+t$ boundary containing neighboring boron atoms is lower in energy than the $t+i$ boundary where boron atoms are well separated.

The structure of free (310) surfaces with segregated boron was obtained by replacing copper with boron in the top lay- 

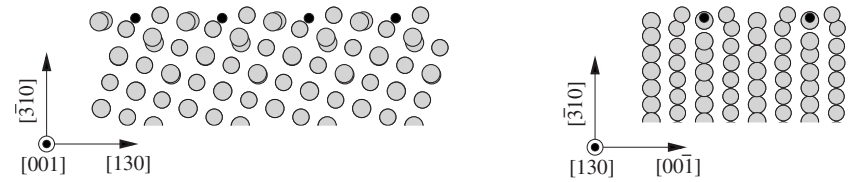

FIG. 7. Two side views of the relaxed $\mathrm{Cu}(310)$ surface with 0.5 ML of boron. Boron atoms were initially placed instead of the top layer of $\mathrm{Cu}$ atoms from which every other atom along the [001] direction was removed. During the relaxation, boron atoms descend below the next $\mathrm{Cu}$ layer causing a noticeable distortion of the latter.

er(s) and allowing the surface to relax. For fractional coverages 0.5 and $1.5 \mathrm{ML}$, we tried either to substitute half of the host atoms in a layer with impurity (substitutional positions) or to place half a monolayer of impurity atoms above the top surface layer (adatom positions). The latter resulted in lower energy configurations.

During relaxation, boron atoms embed into the substrate, often going beneath the top copper layer. As a result, the top copper layer becomes strongly distorted. Figure 7 shows the $\mathrm{Cu}(310)$ surface with $0.5 \mathrm{ML}$ of $\mathrm{B}$ (and 0.5 ML of vacancies) in the first layer. The plane of B atoms indeed resides below the top layer of $\mathrm{Cu}$ atoms and just slightly above the second layer, whereas the host atoms in the surface layer are strongly displaced toward the nearest boron atom.

\section{Reasons behind grain boundary strengthening}

We now apply the ghost impurity cycle to the grain boundaries described in the previous two subsections in order to understand why boron segregation has a positive effect on $W_{\text {sep }}$. Contributions from the HR, SS, and CC mechanisms defined in Sec. I in terms of the work of separation and segregation energies are listed in Tables IV and V, respectively. We choose to evaluate the segregation energies in Table $\mathrm{V}$, assuming interstitial boron $\mathrm{B}_{i}$ to be the bulk ground state. With this choice, we can directly compare contributions to the SS mechanism to those obtained in other studies
(Table VI). The segregation energies for configurations $\mathbf{C}$ and $\mathbf{D}$ become just energies required to create unrelaxed vacancies at an interface taken with opposite signs (our convention here and in Ref. 8 is that the bulk impurity is always fully relaxed, whether this is a real impurity or a vacancy: relaxed "interstitial vacancy" is just a perfect bulk). If one wants to change to boron dumbbells $\mathrm{B}_{d}$, then the segregation energies in Table $\mathrm{V}$ should be modified as follows: $E_{\text {seg }}$ for configuration $\mathbf{B}$ decreases by $0.78 \mathrm{eV}$ (the difference between the enthalpies of solution of $\mathrm{B}_{i}$ and $\mathrm{B}_{d}$ ) and $E_{\text {seg }}$ for configurations $\mathbf{C}$ and $\mathbf{D}$ decrease by $1.27 / 2 \mathrm{eV}$ (half the vacancy formation enthalpy in pure bulk, Ref. 8). Works of separation in Table IV do not depend on the bulk reference, as it cancels out in Eq. (2).

Intuitively, one may expect that the segregation of boron to interstitial sites at the $0.5 \mathrm{ML}$ coverage would reinforce the boundary because additional atoms lead to additional cohesion across the interface, provided that the boundary is not much distorted. Table IV supports this expectation as the total increase in $W_{\text {sep }}$ by $0.46 \mathrm{~J} / \mathrm{m}^{2}$ is provided almost exclusively by the CC mechanism. The SS contribution is also positive but small, whereas the HR contribution for interstitial impurity is zero by definition. Two other $0.5 \mathrm{ML}$ configurations, $l$ and $t$, do not increase $W_{\text {sep }}$ because of the large negative HR contribution that arises if boron replaces host atoms.

A similar effect of interstitial boron is found in ab initio studies of grain boundaries in $\mathrm{Fe}, \mathrm{Ni}$, and $\mathrm{Mo}$ (see Table VI). Boron improves the cohesion at all grain boundaries, and this is mostly due to the CC mechanism. The SS contribution enhances grain boundary strength even more despite the surface and grain boundary terms being themselves negative; i.e., boron distorts free surfaces more than grain boundaries.

As boron segregation proceeds beyond 0.5 ML, the boundary is still strengthened although the magnitude of the effect is diminished. Higher coverage configurations necessarily include the removal of host atoms since all interstitial sites are already filled at 0.5 ML. Hence, the mechanism of strengthening is different.

TABLE IV. Work of separation $W_{\text {sep }}$ at the vertices of the ghost impurity cycle (Fig. 1) and its change $\Delta W_{\text {sep }}$ due to transitions between the vertices. The latter have the meaning of the contributions of the SS, HR, and CC mechanisms. The positive sign of $\Delta W_{\text {sep }}$ corresponds to cohesion enhancement.

\begin{tabular}{|c|c|c|c|c|c|c|c|c|c|c|}
\hline \multirow[b]{2}{*}{ Impurity } & \multirow[b]{2}{*}{ Site } & \multirow{2}{*}{$\begin{array}{c}\text { Excess } \\
(\mathrm{ML})\end{array}$} & \multicolumn{4}{|c|}{$\begin{array}{c}W_{\text {sep }} \\
\left(\mathrm{J} / \mathrm{m}^{2}\right)\end{array}$} & \multicolumn{4}{|c|}{$\begin{array}{l}\Delta W_{\text {sep }} \\
\left(\mathrm{J} / \mathrm{m}^{2}\right)\end{array}$} \\
\hline & & & A & B & $\mathrm{C}$ & $\mathrm{D}$ & Total & SS & HR & $\mathrm{CC}$ \\
\hline \multirow[t]{7}{*}{ B } & $i$ & 0.5 & 3.35 & 3.81 & 3.38 & 3.35 & 0.46 & 0.03 & 0 & 0.43 \\
\hline & $l$ & 0.5 & 3.35 & 2.49 & 2.20 & 2.14 & -0.86 & 0.06 & -1.21 & 0.29 \\
\hline & $t$ & 0.5 & 3.35 & 3.35 & 2.87 & 1.92 & 0.00 & 0.95 & -1.43 & 0.48 \\
\hline & $l+t$ & 1.0 & 3.35 & 3.57 & 3.08 & 1.35 & 0.22 & 1.73 & -2.00 & 0.49 \\
\hline & $l+i$ & 1.0 & 3.35 & 3.24 & 2.84 & 2.18 & -0.11 & 0.66 & -1.17 & 0.40 \\
\hline & $t+i$ & 1.0 & 3.35 & 3.21 & 3.15 & 1.96 & -0.14 & 1.19 & -1.39 & 0.06 \\
\hline & $l+t+i$ & 1.5 & 3.35 & 3.58 & 3.33 & 1.38 & 0.23 & 1.95 & -1.97 & 0.25 \\
\hline \multirow[t]{2}{*}{$\mathrm{Bi}$} & $l$ & 0.5 & 3.35 & 2.15 & 1.81 & 2.20 & -1.20 & -0.39 & -1.15 & 0.34 \\
\hline & $l+t$ & 1.0 & 3.35 & 0.96 & 0.13 & 1.38 & -2.39 & -1.25 & -1.97 & 0.83 \\
\hline
\end{tabular}


TABLE V. Surface and grain boundary segregation energies $E_{\text {seg }}$ at the vertices of the ghost impurity cycle (Fig. 1) and their change $\Delta E_{\text {seg }}$ due to transitions between the vertices. $E_{\text {seg }}$ are defined as average segregation energies per impurity atom (or vacancy), and the sign convention is that positive $E_{\text {seg }}$ means that impurity wants to segregate. Segregation energies for the pure boundary $E_{\text {seg }}(\mathrm{A})$ are set to zero. The bulk reference used here is an interstitial impurity $\mathrm{B}_{i}$ not a dumbbell $\mathrm{B}_{d}$ as in Table III.

\begin{tabular}{|c|c|c|c|c|c|c|c|c|c|c|c|c|c|c|c|c|}
\hline \multirow[b]{3}{*}{ Impurity } & \multirow[b]{3}{*}{ Site } & \multirow{3}{*}{$\begin{array}{c}\text { Excess } \\
(\mathrm{ML})\end{array}$} & \multicolumn{6}{|c|}{$\begin{array}{l}E_{\mathrm{seg}} \\
(\mathrm{eV})\end{array}$} & \multicolumn{8}{|c|}{$\begin{array}{c}\Delta E_{\mathrm{seg}} \\
(\mathrm{eV})\end{array}$} \\
\hline & & & \multicolumn{2}{|c|}{ B } & \multicolumn{2}{|c|}{$\mathrm{C}$} & \multicolumn{2}{|c|}{$\mathrm{D}$} & \multicolumn{2}{|c|}{ Total } & \multicolumn{2}{|c|}{ SS } & \multicolumn{2}{|c|}{ HR } & \multicolumn{2}{|c|}{$\mathrm{CC}$} \\
\hline & & & surf & $\mathrm{gb}$ & surf & $\mathrm{gb}$ & surf & $\mathrm{gb}$ & surf & $\mathrm{gb}$ & surf & $\mathrm{gb}$ & surf & $\mathrm{gb}$ & surf & $\mathrm{gb}$ \\
\hline \multirow[t]{7}{*}{ B } & $i$ & 0.5 & 1.18 & 1.74 & -0.14 & -0.10 & 0 & 0 & 1.18 & 1.74 & -0.14 & -0.10 & 0 & 0 & 1.32 & 1.84 \\
\hline & $l$ & 0.5 & 1.18 & 0.12 & -0.14 & -1.56 & 0 & -1.49 & 1.18 & 0.12 & -0.14 & -0.07 & 0 & -1.49 & 1.32 & 1.68 \\
\hline & $t$ & 0.5 & 1.18 & 1.18 & -0.14 & -0.73 & 0 & -1.76 & 1.18 & 1.18 & -0.14 & 1.03 & 0 & -1.76 & 1.32 & 1.91 \\
\hline & $l+t$ & 1.0 & 1.16 & 1.29 & -0.34 & -0.50 & -0.02 & -1.25 & 1.16 & 1.29 & -0.32 & 0.75 & -0.02 & -1.25 & 1.50 & 1.79 \\
\hline & $l+i$ & 1.0 & 1.16 & 1.09 & -0.34 & -0.65 & -0.02 & -0.74 & 1.16 & 1.09 & -0.32 & 0.09 & -0.02 & -0.74 & 1.50 & 1.74 \\
\hline & $t+i$ & 1.0 & 1.16 & 1.07 & -0.34 & -0.46 & -0.02 & -0.88 & 1.16 & 1.07 & -0.32 & 0.42 & -0.02 & -0.88 & 1.50 & 1.53 \\
\hline & $l+t+i$ & 1.5 & 1.15 & 1.25 & -0.41 & -0.42 & -0.03 & -0.84 & 1.15 & 1.25 & -0.38 & 0.42 & -0.03 & -0.84 & 1.56 & 1.67 \\
\hline \multirow[t]{2}{*}{$\mathrm{Bi}$} & $l$ & 0.5 & 3.11 & 1.63 & 1.18 & -0.71 & 1.20 & -0.22 & 3.11 & 1.63 & -0.02 & -0.49 & 1.20 & -0.22 & 1.93 & 2.34 \\
\hline & $l+t$ & 1.0 & 3.01 & 1.54 & 1.23 & -0.75 & 1.22 & 0.02 & 3.01 & 1.54 & 0.01 & -0.77 & 1.22 & 0.02 & 1.78 & 2.29 \\
\hline
\end{tabular}

To understand this mechanism, it is instructive to compare boron results to those for bismuth, ${ }^{8}$ which are reproduced in the tables for convenience. A striking difference between B and $\mathrm{Bi}$ (or other oversized impurities studied in Ref. 8) is the sign of the SS contribution. The SS mechanism always increases $W_{\text {sep }}$ for boron (Table IV) and decreases $W_{\text {sep }}$ for $\mathrm{Bi}$, $\mathrm{Na}$, and even Ag without any exception. Compare, for example, boron and bismuth at the loose site $(0.5 \mathrm{ML}$ coverage). In both cases, the boundary is weakened, more by $\mathrm{Bi}$, less by $\mathrm{B}$. The negative HR contributions are similar; the $\mathrm{CC}$ mechanism acts so as to strengthen the boundary and is even more efficient for Bi than for B. Thus, it is the SS mechanism which makes the difference, being positive for B but negative for Bi. The loose site is not, of course, the best choice for boron, but even here it is much less harmful than $\mathrm{Bi}$. The comparison of $\mathrm{B}$ and $\mathrm{Bi}$ for the $1 \mathrm{ML} l+t$ case is even more telling. The HR mechanism again has large detrimental effect for both the CC mechanism acts in the opposite direction and is nearly twice as large for $\mathrm{Bi}$ than for $\mathrm{B}$. Finally, the negative SS contribution is large enough to make the boundary brittle in the Bi case, ${ }^{8}$ but the large and positive SS contribution for B results in the boundary being strengthened. In other words, the difference in the distortion pattern of grain boundaries and surfaces is itself sufficient to either strengthen the boundary or to make it brittle.

What are the reasons for the SS contribution being positive for boron? Let us analyze surface and grain boundary contributions for configurations $l$ and $l+t$ for $\mathrm{B}$ and $\mathrm{Bi}$ in Table V. The surface contribution to the SS mechanism for $\mathrm{Bi}$ is negligible; therefore, the negative (embrittling) effect comes from the grain boundary distortion. For boron, this differs in two ways. First, there is always a negative surface term, and, second, the grain boundary term can be large and positive. Even if the latter is negative (as in cases $l$ and $i$ ), it is still smaller than the surface, which renders their difference positive.

TABLE VI. Boron impurity at an interstitial grain boundary site in different materials: contributions of the SS and CC mechanisms in terms of the difference of segregation energies $E_{\mathrm{seg}}^{\mathrm{gb}}-E_{\mathrm{seg}}^{\mathrm{s}}$ (in $\mathrm{eV}$ per impurity atom). For the SS mechanism, individual surface and grain boundary contributions, $E_{\mathrm{seg}}^{\mathrm{s}}$ and $E_{\mathrm{seg}}^{\mathrm{gb}}$, are also shown. The energies in Refs. 36-40 are defined through the difference of binding rather than segregation energies. The results, however, can be directly compared. Note that the SS and CC mechanisms in Refs. 39 and 40 are referred to as the mechanical and chemical contributions, respectively.

\begin{tabular}{|c|c|c|c|c|c|c|c|c|c|}
\hline \multirow[b]{2}{*}{ Host } & \multirow[b]{2}{*}{ Grain boundary } & \multirow[b]{2}{*}{ Method } & \multirow{2}{*}{$\frac{\text { Total }}{\mathrm{gb}-\mathrm{surf}}$} & \multicolumn{3}{|c|}{ SS } & \multirow{2}{*}{$\frac{\mathrm{CC}}{\mathrm{gb}-\mathrm{surf}}$} & \multirow{2}{*}{$\begin{array}{l}\text { Cohesion } \\
\text { enhancer? }\end{array}$} & \multirow[b]{2}{*}{ Ref. } \\
\hline & & & & surf & $\mathrm{gb}$ & $\mathrm{gb}-\operatorname{surf}$ & & & \\
\hline $\mathrm{Fe}$ & $\Sigma 5(010)[001]$ & DMol (LDA) & 1.96 & & & & & Yes & 36 \\
\hline $\mathrm{Fe}$ & $\Sigma 5(210)[001]$ & USPP (LDA) & 0.49 & & & & & Yes & 37 \\
\hline $\mathrm{Fe}$ & $\Sigma 3(111)[1 \overline{1} 0]$ & FLAPW(LDA) & 1.07 & & & & & Yes & 38 \\
\hline $\mathrm{Ni}$ & $\Sigma 5(210)[001]$ & FLAPW (GGA) & 0.49 & -0.27 & -0.16 & 0.11 & 0.38 & Yes & 39 \\
\hline Mo & $\Sigma 5(310)[001]$ & MBPP (LDA) & 2.09 & -0.95 & -0.23 & 0.71 & 1.37 & Yes & 40 \\
\hline $\mathrm{Cu}$ & $\Sigma 5(310)[001]$ & FP LMTO (LDA) & 0.56 & -0.14 & -0.10 & 0.04 & 0.52 & Yes & $\begin{array}{c}\text { present } \\
\text { study }\end{array}$ \\
\hline
\end{tabular}


It is easy to see why the surface contribution to SS is negative for boron. It indicates a sizable distortion of the surface region and arises for impurities that can embed themselves into surface layers (Sec. V B). This would be possible for small impurities, especially if they prefer interstitial positions in the bulk.

It is less obvious why the grain boundary contribution to SS tends to be positive. The SS contribution in the ghost impurity cycle is the energy change when a pure grain boundary with preinserted unrelaxed vacancies $\mathbf{D}$ is further deformed as prescribed by impurity ghosts to arrive at configuration C. Atoms in configuration D would want to relax toward the vacancies, whereas deformation corresponding to large impurity atoms forces them to move further away. The total energy increases and the SS contribution is negative (embrittling). For small substitutional impurities, this is reversed-during the $\mathbf{D} \rightarrow \mathbf{C}$ transition, atoms move toward the vacancies. Hence, the energy decreases and SS is positive (cohesion enhancing). If boron occupies both interstitial and substitutional sites, atomic displacements are more complex. However, the fact that the grain boundary excess volume is always smaller than that of the ideal $\mathrm{Cu}$-like impurity (Fig. 5 ) indicates that, on average, the grain boundary shrinks rather than expands.

The above reasoning relies only on the property of boron atoms being "smaller" than host atoms and therefore seems applicable to other undersize impurities, at least for the light metalloid impurities. Boron was found to reinforce grain boundaries in all materials studied (Table VI). Carbon segregation increases ${ }^{40,41}$ or slightly decreases $W_{\text {sep }},{ }^{37}$ whereas $\mathrm{H}$, $\mathrm{N}$, and $\mathrm{O}$ weaken grain boundaries. ${ }^{39,40,42}$ In the latter case, the embrittling propensity is due to the CC contribution, which becomes large and negative. Janisch and Elsässer ${ }^{40}$ suggested that this should be the case for light species whose outer electronic shell $(1 s$ for $\mathrm{H}$ and $2 p$ for $\mathrm{N}$ and $\mathrm{O}$ ) falls near or below the bottom of the valence band of the host metal.

Negative CC indicates that the insertion of an impurity atom into a prepared hole (configuration $\mathbf{C}$ ) weakens atomic bonds across the interface. This could be the case if the impurity affects the bonds between the neighboring host atoms by means of withdrawing the electronic charge from themwhich is known as the electronic mechanism of embrittlement. An alternative explanation, advocated in Ref. 40, is Cottrell's 43 "unified theory," which refers to the position of the impurity levels relative to the Fermi energy of the host metal. According to this theory, interstitial impurities whose valence electrons lie close to the Fermi level would form predominantly covalent bonds with host atoms and, hence, prefer grain boundaries over surfaces due to a higher coordination in the former (the Cottrell $\sqrt{z}$ factor). This means positive CC and cohesion enhancement. On the other hand, impurities with valence states lying high above or deeply below the Fermi level would form polar bonds with the host atoms and turn into screened ions, for which the surface environment is more favorable. This results in a negative CC contribution, which weakens the boundary.

In our recent calculations of $\mathrm{Cu}$ grain boundary with inert gas atom impurities ( $\mathrm{He}$ and $\mathrm{Kr}$ ), we also observed a large negative $\mathrm{CC}$ contribution leading to catastrophic embrittlement. ${ }^{44}$ As no charge transfer to or from an inert gas atom is expected, the embrittling effect in this case must be related to the Pauli exclusion principle. One may, therefore, hypothesize that a similar mechanism can act for impurities with a nearly completed $p$ shell, such as fluorine, oxygen, and, to a lesser extent, nitrogen, as filling the impurity shells with metal electrons would effectively render the dopant atoms inert-gas-like. The question as to how significant this "inert gas atom" mechanism is in comparison to others requires a separate investigation and is outside the scope of the present study.

\section{CONCLUSIONS}

We have made calculations of boron in bulk and at the (310) surface and $\Sigma 5(310)$ [001] grain boundary in copper. We should emphasize that all three play an important role in determining the grain boundary fracture behavior.

(1) We find that B strengthens the boundary in the whole range of coverages studied (up to $1.5 \mathrm{ML}$ ), with the maximal effect achieved at 0.5 ML. Combined with the observed ability of B to remove harmful impurities, such as $\mathrm{Sb}$ from the copper boundary, ${ }^{4}$ this makes boron a particularly attractive alloying addition.

(2) The reasons behind grain boundary strengthening at 0.5 ML and higher coverages are different. 0.5 ML corresponds to all interstitial positions at the boundary being filled by boron atoms, providing therefore additional cohesion between the grains while not distorting the boundary much (the CC mechanism).

(3) At 1 and $1.5 \mathrm{ML}$, boron begins to substitute host atoms at the boundary, leading to significant distortions and lateral translations of the grains. The SS contribution, however, remains positive and acts so as to increase $W_{\text {sep }}$. We demonstrate that the difference in the sign of the SS contribution proves to be solely responsible for the opposite effect of B compared to embrittling species such as Bi.

(4) Distortion of a free surface by segregated boron atoms further increases $W_{\text {sep }}$, but it is not a decisive factor.

(5) Introducing boron into bulk $\mathrm{Cu}$ leads to a peculiar situation in which substitutional and interstitial impurities are rather close in energy. Combined together, they form a strongly bound dimer held by elastic forces of the host lattice. Remarkably, the heat of solution of the lowest energy $s\langle 100\rangle$ dumbbell (per dimer) is also close to the heat of solution of boron single impurities (per atom). Thus, a sizable proportion of boron atoms should be found in a bound state in most experimental conditions, even at high temperatures.

(6) A large discrepancy between calculated heats of solution and experimental estimations for terminal solubility of B in $\mathrm{Cu}$ is discovered. We are inclined to think that the solubility limits suggested in Ref. 35 and then translated into the existing $\mathrm{B}-\mathrm{Cu}$ phase diagram are overestimated by a few orders of magnitude and hope that our findings inspire experimental work on the updated version of the phase diagram.

\section{ACKNOWLEDGMENTS}

This work has benefited from salutory discussions with 
M. W. Finnis, to whom we are also grateful for a number of valuable suggestions for the manuscript. Useful comments from M. van Schilfgaarde and P. Ballone are much appreciated. We thank T. P. C. Klaver for an independent PAW calculation of pure boron and boron impurity in bulk copper.

\section{APPENDIX: EQUILIBRIUM CONCENTRATION OF COEXISTING IMPURITY TYPES IN AN IDEAL SOLUTION}

We describe a thermodynamic approach, which we use in the paper to estimate the equilibrium concentration of boron single impurities $\left(\mathrm{B}_{i}\right.$ and $\left.\mathrm{B}_{s}\right)$ and dimers $\left(\mathrm{B}_{d}\right)$ in bulk $\mathrm{Cu}$. The approach follows the canonical treatment of ideal solid solutions proposed in Ref. 45 and is applicable to any binary system in which the heats of solution of the impurity species occupying an interstitial position, a substitutional position, or forming a dumbell are comparable.

The reader may be surprised by the fact that the model outlined below ignores thermal vacancies altogether. Indeed, at first glance, this looks inconsistent, given that the heats of solution of boron in $\mathrm{Cu}$ listed in Table II are comparable to the vacancy formation enthalpy $H_{f}^{v}=1.27 \mathrm{eV} .{ }^{8}$ We deliberately omit vacancies as the equilibrium impurity concentrations do not depend on the vacancy formation enthalpy; hence, they do not change even if no vacancies at all are allowed. Indeed, if a crystal contains thermal vacancies with concentration $c_{v}$, an additional term will appear in Eqs. (A4), (A5), and (A7) and, consequently, one more relation will be added to the system of Eqs. (A8). However, $c_{v}$ can be eliminated from Eqs. (A8), explicitly leading to exactly the same set of equations [Eqs. (A9)-(A11)] as below. The equilibrium impurity concentrations, obtained as the solution of Eqs. (A9)-(A11), will therefore not depend on the vacancy concentration either. (Note in passing that the reverse is not true, i.e., the equilibrium concentration of vacancies does depend on impurity concentrations.)

\section{Variables and definitions}

Consider a large piece of crystal $A_{1-x} B_{x}$ containing $N$ lattice sites with $n_{a}$ host atoms $A$ and $n_{b}$ impurity atoms $B$. The latter, in turn, include $n_{s}$ substitutional impurities, $n_{i}$ interstitial impurities, and $n_{d}$ dumbbells,

$$
n_{s}+n_{i}+2 n_{d}=n_{b}
$$

If the crystal is sufficiently large so that any surface effects can be neglected, the resulting equilibrium concentrations should depend on $n_{a}$ and $n_{b}$ only through the composition of the solid solution

$$
x=\frac{n_{b}}{n_{a}+n_{b}} .
$$

Concentrations of host atoms $\left(c_{a}\right)$ and impurity of any type $\left(c_{s}, c_{i}\right.$, and $\left.c_{d}\right)$, defined "per lattice site" here, must satisfy the following two constraints:

$$
N c_{a}=n_{a},
$$

$$
N\left(c_{s}+c_{i}+2 c_{d}\right)=n_{b},
$$

indicating that the total number of atoms of each species is conserved. In addition, we have the "site balance" condition as every lattice position should be occupied by either a host atom, an impurity atom, or an impurity dumbbell,

$$
c_{a}+c_{s}+c_{d}=1 .
$$

Overall, there are five variables $\left(N, c_{a}, c_{s}, c_{i}\right.$, and $\left.c_{d}\right)$ and three constraints [Eqs. (A2)-(A4)]; hence, the system has two degrees of freedom.

If defects do not interact, the total energy of the system is linear in defect concentrations,

$$
E=N\left(c_{a} \varepsilon_{a}+c_{s} \varepsilon_{s}+c_{i} \varepsilon_{i}+2 c_{d} \varepsilon_{d}\right),
$$

where $\varepsilon_{a}$ is the energy (per atom) of the pure crystal, whereas $\varepsilon_{s}, \varepsilon_{i}$, and $\varepsilon_{d}$ are the energies per impurity atom defined by Eq. (A5). In practice, these are usually found from the total energy calculation of supercells containing a single defect of each type. Enthalpies of solution $H_{s}$, such as those listed in Table II, are related to the $\varepsilon$ 's in a simple way,

$$
H_{s}^{i}=\varepsilon_{i}-\varepsilon_{b}, \quad H_{s}^{s}=\varepsilon_{s}-\varepsilon_{b}, \quad H_{s}^{d}=\varepsilon_{d}-\varepsilon_{b},
$$

where $\varepsilon_{b}$ denotes the energy per atom of species $B$ in its pure state.

At zero pressure, the Gibbs free energy of the crystal is

$$
G=E-T S,
$$

where $T$ is the temperature and $S$ is the (configurational) entropy given by

$$
\begin{aligned}
S= & -k N\left[c_{a} \log c_{a}+c_{s} \log c_{s}+c_{d} \log c_{d}-c_{d} \log \eta+c_{i} \log c_{i}\right. \\
& \left.+\left(\alpha-c_{i}\right) \log \left(\alpha-c_{i}\right)-\alpha \log \alpha\right],
\end{aligned}
$$

where $k$ is the Boltzmann constant, $\eta$ is the number of equivalent orientations of the dumbbell, and $\alpha$ is the number of interstitial sites per one lattice site. For the octahedral interstices in the fcc lattice, $\alpha=1$, whereas $\eta=3$ for the $\langle 100\rangle$ dumbbell in cubic crystals. Equations (A5) and (A7) do not take atomic vibrations into account, but these can be easily included, for example, at the level of quasiharmonic approximation. ${ }^{46}$

\section{Equilibrium concentrations}

The equilibrium impurity concentrations are those that minimize the Gibbs free energy [Eq. (A6)] subject to constraints (A2)-(A4). The minimization leads to the following system of five equations:

$$
\begin{array}{r}
G-\mu_{a} n_{a}-\mu_{b} n_{b}=0, \\
\varepsilon_{a}+k T\left(1+\log c_{a}\right)-\mu_{a}-\lambda / N=0, \\
\varepsilon_{s}+k T\left(1+\log c_{s}\right)-\mu_{b}-\lambda / N=0, \\
2 \varepsilon_{d}+k T\left(1+\log c_{d} / \eta\right)-2 \mu_{b}-\lambda / N=0,
\end{array}
$$




$$
\varepsilon_{i}+k T \log \left[c_{i} /\left(\alpha-c_{i}\right)\right]-\mu_{b}=0,
$$

where $\mu_{a}$ and $\mu_{b}$ are Lagrange multipliers associated with Eqs. (A2) and (A3) and therefore have the meaning of the chemical potentials of species $A$ and $B$, respectively. $\lambda$ is the Lagrange coefficient corresponding to Eq. (A4). Equations (A8), together with constraint (A2)-(A4), are sufficient to determine all the unknowns.

The elimination of $\mu_{a}, \mu_{b}$, and $\lambda$ leaves the following two independent relations containing only the impurity concentrations:

$$
\begin{gathered}
\frac{c_{i} / \alpha}{\left(1-c_{i} / \alpha\right)^{\alpha+1} c_{s}}=\exp \left[-\frac{\left(\varepsilon_{i}-\varepsilon_{s}\right)}{k T}\right], \\
\frac{\left(c_{i} / \alpha\right) c_{s}}{\left(1-c_{i} / \alpha\right) c_{d} / \eta}=\exp \left[-\frac{\left(\varepsilon_{i}+\varepsilon_{s}-2 \varepsilon_{d}\right)}{k T}\right],
\end{gathered}
$$

in which the reader might immediately recognize "quasichemical" relations describing defect reactions. Equation (A9), in particular, corresponds to the conversion of a substitutional impurity into an interstitial one, whereas Eq. (A10) describes the dissociation of a dumbbell into an interstitial and a substitutional impurity.

Equations (A9) and (A10), together with the relation

$$
c_{s}+(1-x) c_{i}+(2-x) c_{d}=x,
$$

can be used to find all three concentrations $c_{s}, c_{i}$, and $c_{d}$. [Equation (A11) readily follows from constraints (A2)-(A4) and from the definition of $x$ in Eq. (A1).]

The above consideration applies to an ideal solid solution of arbitrary composition $x$. If the alloy is dilute, $x \ll 1$, finding the solution of Eqs. (A9)-(A11) simplifies and reduces to solving the quadratic

$$
\left[2 \eta e_{d i}^{2}-e_{s i}-\alpha(\alpha+2)\right] z^{2}+\left(e_{s i}+\alpha+2 x\right) z-x=0,
$$

where $e_{d i}$ and $e_{s i}$ denote the following exponentials:

$$
\begin{aligned}
& e_{d i}=\exp \left(-\frac{\varepsilon_{d}-\varepsilon_{i}}{k T}\right)=\exp \left(-\frac{H_{s}^{d}-H_{s}^{i}}{k T}\right), \\
& e_{s i}=\exp \left(-\frac{\varepsilon_{s}-\varepsilon_{i}}{k T}\right)=\exp \left(-\frac{H_{s}^{s}-H_{s}^{i}}{k T}\right) .
\end{aligned}
$$

Equilibrium concentrations are then obtained using the positive root of Eq. (A12), $z_{+}$, as

$$
c_{d}=\eta e_{d i}^{2} z_{+}^{2}, \quad c_{i}=\alpha z_{+}, \quad c_{s}=e_{s d} z_{+} .
$$

\section{Solubility limit}

As pointed out in Ref. 45, the advantage of the above approach is that it produces not only the equilibrium concentrations of defects but also the chemical potentials of the species. The chemical potential of the solute, in particular, can be restored from the equilibrium concentrations $c_{d}, c_{i}$, and $c_{s}$ using any of the following equations:

$$
\begin{aligned}
\mu_{b} & =\varepsilon_{s}+k T\left[\log c_{s}+\alpha \log \left(1-c_{i} / \alpha\right)\right] \\
& =\varepsilon_{i}+k T \log \left[c_{i} /\left(\alpha-c_{i}\right)\right] \\
& =\varepsilon_{d}+\frac{1}{2} k T\left[\log c_{d} / \eta+\alpha \log \left(1-c_{i} / \alpha\right)\right] .
\end{aligned}
$$

These three relations simply express the fact that impurity atoms participating in any of the three types of defects considered here, namely, interstitial impurity, substitutional impurity, and impurity dumbbells, are in equilibrium with each other; hence, their chemical potentials must be equal.

Once the chemical potentials are known, it is straightforward to find the limiting solubilities by considering the equilibrium between the $A$-rich and $B$-rich phases with terminal compositions. The terminal compositions are those that make $\mu_{a}$ and $\mu_{b}$ in both phases equal.

For our purposes, however, it is sufficient to assume that the $B$-rich phase is a pure $B$ crystal, such as the rhombohedral $\alpha$ boron, with $\mu_{b}=e_{b}$. Using again the dilute limit, from Eq. (A14) we obtain the maximal defect concentrations in $A_{1-x} B_{x}$ as

$$
\begin{gathered}
c_{s}^{m}=\exp \left(-\frac{H_{s}^{s}}{k T}\right), \\
c_{i}^{m}=\alpha \exp \left(-\frac{H_{s}^{i}}{k T}\right), \\
c_{d}^{m}=\eta \exp \left(-\frac{2 H_{s}^{d}}{k T}\right) .
\end{gathered}
$$

These, according to Eq. (A11), define the limiting solubility $x^{m}$ as

$$
x^{m}=c_{s}^{m}+c_{i}^{m}+2 c_{d}^{m} .
$$

\section{Results for boron in copper}

Figure 8 shows the equilibrium concentrations of $\mathrm{B}_{i}, \mathrm{~B}_{s}$, and $\mathrm{B}_{d}$ at $T=1000 \mathrm{~K}$ as a function of the boron content $x$. These were obtained using Eqs. (A12) and (A13) with $\alpha=1$, $\eta=3$, and the enthalpies of solution $H_{s}$ listed in Table II (those calculated in a 108 atom supercell). The limiting solubility $x^{m}$ given by Eqs. (A15) and (A16) is shown with a vertical dotted line.

According to Fig. 8, the dumbbells strongly prevail at and above $x^{m}$. (Supersaturated solutions may arise if the excess boron precipitates as some metastable phase, higher in energy than the rhombohedral $\alpha$-B. Such a scenario, however, is not supported by experimental observations ${ }^{27}$ ). With decreasing $x$, the loss of configurational entropy should eventually overweigh the energetic advantage of forming dumbbells, giving rise to the crossover between concentrations of dumbbells and single impurities. This is indeed observed in Fig. 8, although the crossover concentrations seem too small to be experimentally detectable.

Figure 9 shows the temperature dependence of the impurity concentrations at fixed $x$ in the form of the Arrhenius 


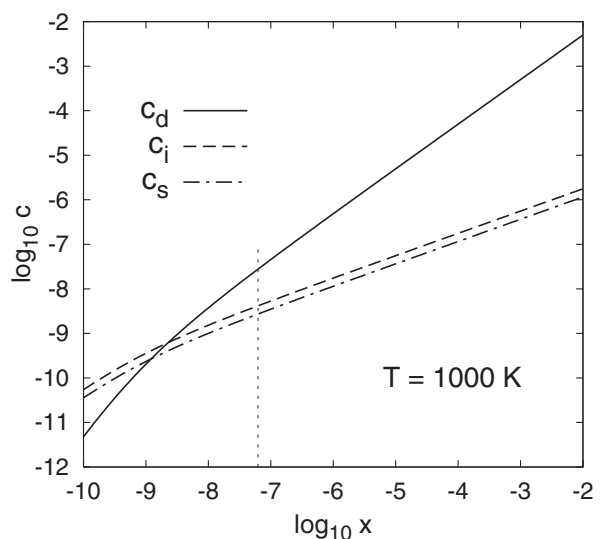

FIG. 8. Equilibrium concentration of $\mathrm{B}_{d}, \mathrm{~B}_{i}$, and $\mathrm{B}_{s}$ in $\mathrm{Cu}_{1-x} \mathrm{~B}_{x}$ at $T=1000 \mathrm{~K}$ given by Eqs. (A12) and (A13) as a function of boron content $x$. The vertical dotted line corresponds to the solubility limit $x^{m}$ estimated according to Eq. (A16).

plot $\log c=f(1 / T)$. Concentration $x$ for this plot is chosen as the limiting solubility in Fig. 8. As a result, the curves in Fig. 9 have a kink at $T=1000 \mathrm{~K}$, which corresponds to the precipitation of the second phase. The curves to the left of the kink are the equilibrium defect concentrations in a single phase crystal $\mathrm{Cu}_{1-x} \mathrm{~B}_{x}$ given by Eqs. (A12) and (A13), whereas concentrations to the right of the kink correspond to a two-phase equilibrium between $\mathrm{Cu}_{1-x} \mathrm{~B}_{x}$ and pure $\alpha$-boron. The latter are given by Eq. (A15) and are just straight lines in the Arrhenius coordinates.

We again observe the dominance of boron dumbbells in the whole range of temperatures, except the narrow region close to the eutectic temperature $T_{e}=1013{ }^{\circ} \mathrm{C}$ (vertical dotted line in Fig. 9). The crossover point appears here for the same reason as in Fig. 8 and rapidly moves to higher temperatures with increasing $x$. If, for example, for this plot we use $x$ corresponding to the limiting solubility at $T_{e}$, then the crossover would move above both the eutectic temperature and melting temperature of pure copper $T_{m}=1085{ }^{\circ} \mathrm{C}$, so the dumbbells would dominate everywhere.

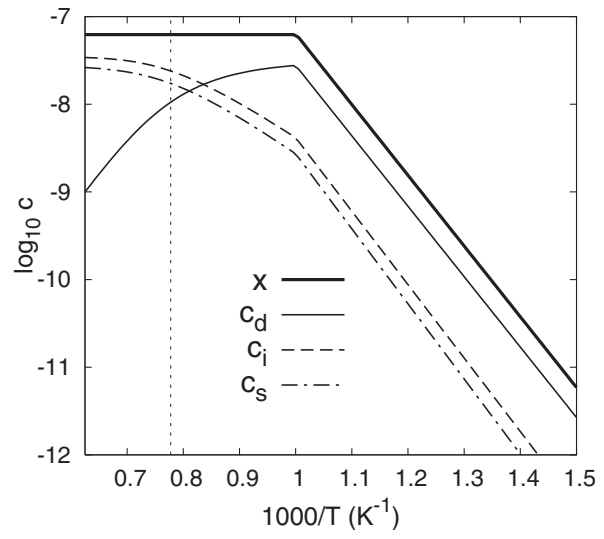

FIG. 9. Equilibrium concentration of $\mathrm{B}_{d}, \mathrm{~B}_{i}$, and $\mathrm{B}_{s}$ and the total amount of boron, $x=c_{i}+c_{s}+2 c_{d}$, as a function of $T^{-1}$ (the Arrhenius plot). The alloy composition is taken as the terminal solution at $T$ $=1000 \mathrm{~K}$ (vertical dotted line in Fig. 8). The kink on the curves corresponds to the precipitation of the second phase (assumed to be pure $\alpha-\mathrm{B})$. The vertical dotted line indicates the eutectic temperature $T_{e}=1013{ }^{\circ} \mathrm{C}$.

To summarize, we observe that boron dumbbells $\mathrm{B}_{d}$ are the essential component of $\mathrm{Cu}_{1-x} \mathrm{~B}_{x}$ solid solutions both in the single phase and two phase regions of the $\mathrm{Cu}-\mathrm{B}$ phase diagram. As a matter of fact, the concentration of boron dumbbells $\mathrm{B}_{d}$ exceeds those of single impurities $\mathrm{B}_{i}$ and $\mathrm{B}_{s}$ in most conditions. High temperatures and small boron contents tend to make these concentrations comparable at best. The dumbbells can be suppressed only in very diluted samples where the impurity concentrations are likely to fall below the detection limit anyway.

The terminal solubility of $\mathrm{B}$ in $\mathrm{Cu}$ appears to be lower than that indicated in published phase diagrams. ${ }^{3}$ It is of the order of a few ppm at $T_{e}(0.29$ at. \% in Ref. 3$)$ and is as low as $10^{-29}$ at room temperature (0.06 at. \% in Ref. 13$)$. The fact that the second phase precipitates as a solid solution of $\mathrm{Cu}$ in $\mathrm{B},{ }^{3,27}$ rather than as a pure boron (assumed here) leads to even lower limiting solubilities.
${ }^{1}$ C. T. Liu, C. L. White, and J. A. Horton, Acta Metall. 33, 213 (1985)

${ }^{2}$ P. Lejček and A. Fraczkiewicz, Intermetallics 11, 1053 (2003).

${ }^{3}$ Binary Alloy Phase Diagrams, 2nd ed., edited by T. B. Massalski (ASM International, Materials Park, OH, 1996).

${ }^{4}$ E. E. Glikman, Y. V. Goryunov, and A. M. Zherdev, Sov. Phys. J. 17, 946 (1974).

${ }^{5}$ R. Suryanarayanan, C. A. Frey, S. M. L. Sastry, B. E. Waller, S. E. Bates, and W. E. Buhro, Mater. Sci. Eng., A 264, 210 (1999).

${ }^{6}$ D. E. Ellis, K. C. Mundim, D. Fuks, S. Dorfman, and A. Berner, Philos. Mag. B 79, 1615 (1999).

${ }^{7}$ M. Füllgrabe, B. Ittermann, H.-J. Stöckmann, F. Kroll, D. Peters, and H. Ackermann, Phys. Rev. B 64, 224302 (2001).

${ }^{8}$ A. Y. Lozovoi, A. T. Paxton, and M. W. Finnis, Phys. Rev. B 74, 155416 (2006).

${ }^{9}$ J. R. Rice and R. Thomson, Philos. Mag. 29, 73 (1974).
${ }^{10}$ J. R. Rice and J.-S. Wang, Mater. Sci. Eng., A 107, 23 (1989).

${ }^{11}$ J. Donohue, The Structures of The Elements (Wiley, New York, 1974).

${ }^{12}$ C. Mailhiot, J. B. Grant, and A. K. McMahan, Phys. Rev. B 42, 9033 (1990).

${ }^{13}$ T. W. Barbee, A. K. McMahan, J. E. Klepeis, and M. van Schilfgaarde, Phys. Rev. B 56, 5148 (1997).

${ }^{14}$ S. Lee, D. M. Bylander, and L. Kleinman, Phys. Rev. B 42, 1316 (1990).

${ }^{15}$ N. Vast, S. Baroni, G. Zerah, J. M. Besson, A. Polian, M. Grimsditch, and J. C. Chervin, Phys. Rev. Lett. 78, 693 (1997).

${ }^{16}$ A. Masago, K. Shirai, and H. Katayama-Yoshida, Phys. Rev. B 73, 104102 (2006).

${ }^{17}$ D. L. V. K. Prasad, M. M. Balakrishnarajan, and E. D. Jemmis, Phys. Rev. B 72, 195102 (2005).

${ }^{18}$ U. Häussermann, S. I. Simak, R. Ahuja, and B. Johansson, Phys. 
Rev. Lett. 90, 065701 (2003).

${ }^{19}$ R. J. Nelmes, J. S. Loveday, D. R. Allan, J. M. Besson, G. Hamel, P. Grima, and S. Hull, Phys. Rev. B 47, 7668 (1993).

${ }^{20}$ M. Methfessel, M. van Schilfgaarde, and R. A. Casali, Lect. Notes Phys. 535, 114 (2000).

${ }^{21}$ U. von Barth and L. Hedin, J. Phys. C 5, 1629 (1972).

${ }^{22}$ V. L. Moruzzi, J. F. Janak, and A. R. Williams, Calculated Electronic Properties of Metals (Pergamon, New York, 1978).

${ }^{23}$ By the impurity ground state, we understand the dominant type of impurity (interstitial, substitutional, etc.) in the single phase alloy in the $T \rightarrow 0$ limit.

${ }^{24}$ B. Ittermann, H. Ackermann, H.-J. Stöckmann, K.-H. Ergezinger, M. Heemeier, F. Kroll, F. Mai, K. Marbach, D. Peters, and G. Sulzer, Phys. Rev. Lett. 77, 4784 (1996).

${ }^{25}$ H.-J. Stöckmann, K.-H. Ergezinger, M. Füllgrabe, B. Ittermann, F. Kroll, and D. Peters, Phys. Rev. B 64, 224301 (2001).

${ }^{26}$ E. W. Dewing, Metall. Trans. A 21A, 2609 (1990).

${ }^{27}$ J. Rexer and G. Petzow, Metall 24, 1083 (1970).

${ }^{28}$ K. T. Jacob, S. Priya, and Y. Waseda, Metall. Mater. Trans. A 151A, 2674 (2000).

${ }^{29}$ P. E. Blöchl, Phys. Rev. B 50, 17953 (1994).

${ }^{30}$ G. Kresse and D. Joubert, Phys. Rev. B 59, 1758 (1999).

${ }^{31}$ G. Kresse and J. Hafner, Phys. Rev. B 47, 558 (1993).
${ }^{32}$ G. Kresse and J. Furthmüller, Phys. Rev. B 54, 11169 (1996).

${ }^{33}$ J. P. Perdew, K. Burke, and M. Ernzerhof, Phys. Rev. Lett. 77, 3865 (1996).

${ }^{34}$ D. J. Chakrabarti and D. E. Laughlin, Bull. Alloy Phase Diagrams 3, 45 (1982).

${ }^{35}$ A. P. Smiryagin and O. S. Kvurt, Tr. Gos. Nauchn. Issled. Proektn. Inst. Splavov Obrabotki Tsvetn. Metal. 24, 7 (1965).

${ }^{36}$ Z.-Z. Chen and C.-Y. Wang, J. Phys.: Condens. Matter 17, 6645 (2005).

${ }^{37}$ J. S. Braithwaite and P. Rez, Acta Mater. 53, 2715 (2005).

${ }^{38}$ R. Wu, L. P. Zhong, L. J. Chen, and A. J. Freeman, Phys. Rev. B 54, 7084 (1996).

${ }^{39}$ W. T. Geng, A. J. Freeman, R. Wu, C. B. Geller, and J. E. Raynolds, Phys. Rev. B 60, 7149 (1999).

${ }^{40}$ R. Janisch and C. Elsässer, Phys. Rev. B 67, 224101 (2003).

${ }^{41}$ R. Wu, A. J. Freeman, and G. B. Olson, Phys. Rev. B 53, 7504 (1996).

${ }^{42}$ L. Zhong, R. Wu, A. J. Freeman, and G. B. Olson, Phys. Rev. B 62, 13938 (2000).

${ }^{43}$ A. H. Cottrell, Mat. Sci. Technol. 6, 807 (2000).

${ }^{44}$ A. Y. Lozovoi and A. T. Paxton (unpublished).

${ }^{45}$ M. Hagen and M. Finnis, Philos. Mag. A 77, 447 (1998).

${ }^{46}$ A. Y. Lozovoi and Y. Mishin, Phys. Rev. B 68, 184113 (2003). 